\title{
Síndrome de Schmidt: reporte de caso y revisión de literatura
}

\author{
Schmidt Syndrome: A Case Report and Literature Review
}

\author{
González-Macías $N A,{ }^{1}$ Rojas-García $W^{2}$
}

\author{
${ }^{1}$ Médico, semillero de investigación Endocrinología, Fundación \\ Universitaria de Ciencias de la Salud. \\ ${ }^{2}$ Docente, servicio de endocrinología, Hospital de San José, \\ Fundación Universitaria de Ciencias de la Salud. \\ Autor de correspondencia: Natalia Andrea González Macias \\ Correo electrónico: nagonzalez2@fucsalud.edu.co \\ Fecha de recepción: 7/12/2019 \\ Fecha de aceptación: 9/02/2020
}

\section{Resumen}

El síndrome poliglandular autoinmunitario es una entidad clínica caracterizada por la insuficiencia de dos o más glándulas con funciones endocrinas, mediada por mecanismos inmunológicos, de los que se conocen cuatro subtipos en la actualidad. En el presente artículo se presenta el caso de una joven de 16 años con disfunción suprarrenal, tiroidea y gonadal, en la que se diagnostica síndrome de Schmidt después de la evaluación clínica y paraclínica.

Palabras clave: poliendocrinopatías autoinmunes, síndrome poliglandular autoinmune tipo II, síndrome de Schmidt.

\section{Abstract}

Autoimmune polyglandular syndrome is a clinical entity characterized by the insufficiency of two or more glands with endocrine functions, mediated by immunological mechanisms, actually are known 4 subtypes. This article presents a case of a 16-year-old girl with adrenal, thyroid, and gonadal dysfunction in whom after clinical and paraclinical evaluation was diagnosed with Schmidt syndrome.

Keywords: polyendocrinopathies autoimmune, autoimmune polyglandular syndrome type II, Schmidt's syndrome.

\section{Introducción}

El síndrome poliglandular autoinmunitario es una rara entidad, que tiene una incidencia de 1,5 casos por cada
100000 habitantes (1). Su etiología es inmunológica y lleva a la disfunción de al menos dos sistemas glandulares endocrinos. Considerando los subtipos clínicos que posee esta entidad, las manifestaciones clínicas pueden ser variadas y es importante tenerlas en cuenta para realizar un adecuado abordaje diagnóstico y terapéutico. En el presente reporte se expone el caso de una paciente con síndrome poliglandular autoinmunitario tipo II o síndrome de Schmidt.

\section{Presentación de caso}

Paciente femenina de 16 años, llevada al servicio de urgencias del Hospital Universitario Infantil de San José de Bogotá por un cuadro clínico de 8 meses de evolución dado por astenia, adinamia y un día de episodios eméticos de contenido alimentario. Como antecedentes de importancia se registran un hipotiroidismo tratado con levotiroxina, 75 microgramos día, y anemia en suplencia con sulfato ferroso en 300 mg día; además de esto también se informa una hospitalización por hiponatremia hace 1 año. A la revisión por sistemas refiere pérdida de peso de $12 \mathrm{~kg}$ en 1 mes, fenómeno de Raynaud y amenorrea hace 8 meses.

Durante el examen físico se evidenció palidez mucocutánea generalizada, alopecia areata con cuatro lesiones de aproximadamente 2 por $3 \mathrm{~cm}$ en la región parietooccipital, máculas pardo-violáceas que comprometen la región alveolar de reborde incisal superior y el dorso de la lengua, además de livedo reticular en las extremidades, el resto del examen físico estuvo dentro de los parámetros de normalidad. En la Tabla 1 se muestran los resultados de los exámenes de laboratorio.

Se evidencia hiponatremia leve e hiperpotasemia severa, por lo cual se inician medidas para la corrección de los electrolitos. Se solicita cortisol a las 8 a. m. con reporte bajo con respecto al límite de referencia, reporte de ACTH elevado y anticuerpos anti-21-hidroxilasa positivos, por lo que se considera insuficiencia suprarrenal primaria autoinmunitaria y se decide iniciar tratamiento con hidrocortisona $10 \mathrm{mg}$ a las $8 \mathrm{a} . \mathrm{m}$., seguido de una segunda dosis de $5 \mathrm{mg}$ a las $12 \mathrm{~m}$. y una última 
Tabla 1. Exámenes de laboratorio

\begin{tabular}{|c|c|}
\hline Paraclínico & Resultado \\
\hline Leucocitos & 3840 millones $/ \mathrm{mm}^{3}$ (4500-10 000 millones $/ \mathrm{mm}^{3}$ ) \\
\hline Neutrófilos & 3700 millones $/ \mathrm{mm}^{3}$ (2500-7500 millones $/ \mathrm{mm}^{3}$ ) \\
\hline Plaquetas & 417000 millones $/ \mathrm{mm}^{3}\left(150000-450000\right.$ millones $\left./ \mathrm{mm}^{3}\right)$ \\
\hline Hemoglobina & $10 \mathrm{~g} / \mathrm{dL}(12-16 \mathrm{~g} / \mathrm{dL})$ \\
\hline Hematocrito & $28 \%(36,1 \%-44,3 \%)$ \\
\hline Volumen corpuscular medio & $81,9 \mathrm{fl}(80-100 \mathrm{fl})$ \\
\hline CHCM & $35,7 \mathrm{~g} / \mathrm{dL}(36 / 38 \mathrm{~g} / \mathrm{dL})$ \\
\hline Ancho de distribución eritrocitaria & $12,3 \%(14,5 \%-15 \%)$ \\
\hline Hierro sérico & $64 \mu \mathrm{g} / \mathrm{dL}(60-170 \mu \mathrm{g} / \mathrm{dL})$ \\
\hline Transferrina & $21 \mathrm{mg} / \mathrm{dL}(170-290 \mathrm{mg} / \mathrm{dL})$ \\
\hline Ferritina & $234,4 \mathrm{ng} / \mathrm{mL}(20-400 \mathrm{ng} / \mathrm{mL})$ \\
\hline Sodio & $125,5 \mathrm{mEq} / \mathrm{L}(136-145 \mathrm{mEq} / \mathrm{L})$ \\
\hline Potasio & $7,04 \mathrm{mEq} / \mathrm{L}(3,5-5,3 \mathrm{mEq} / \mathrm{L})$ \\
\hline Magnesio & $1,62 \mathrm{mEq} / \mathrm{L}(1,5-2,5 \mathrm{mEq} / \mathrm{L})$ \\
\hline Calcio & $9,1 \mathrm{mg} / \mathrm{dL}(8,5-10,2 \mathrm{mg} / \mathrm{dL})$ \\
\hline Cloro & $103,5 \mathrm{mEq} / \mathrm{L}(97-107 \mathrm{mEq} / \mathrm{L})$ \\
\hline Glucosa & 95 mg/dL (70-100 mg/dL) \\
\hline Nitrógeno ureico & $34,7 \mathrm{mg} / \mathrm{dL}(6-20 \mathrm{mg} / \mathrm{dL})$ \\
\hline Creatinina & $1,3 \mathrm{mg} / \mathrm{dL}(07-1,3 \mathrm{mg} / \mathrm{dL})$ \\
\hline Proteínas totales & $6,8 \mathrm{~g} / \mathrm{dL}(6,0-8,3 \mathrm{~g} / \mathrm{dL})$ \\
\hline Albúmina & $3,8 \mathrm{~g} / \mathrm{dL}(3,4-5,4 \mathrm{~g} / \mathrm{dL})$ \\
\hline Globina & $3,8 \mathrm{~g} / \mathrm{dL}(2,0-3,5 \mathrm{~g} / \mathrm{dL})$ \\
\hline ACTH & $110 \mathrm{pg} / \mathrm{mL}(20-80 \mathrm{pg} / \mathrm{mL})$ \\
\hline TSH & 6,4 mUl/L (0,37-4,7 mUl/L) \\
\hline $\mathrm{T}_{4}$ libre & $1,14 \mu \mathrm{g} / \mathrm{dL}(4,5-11,2 \mu \mathrm{g} / \mathrm{dL})$ \\
\hline Anticuerpos tiroideos antitiroglobulina (ATG) & $112 \mathrm{ng} / \mathrm{mL}(0-40 \mathrm{ng} / \mathrm{mL})$ \\
\hline Anticuerpos antiperoxidasa (TPO) & $125 \mathrm{ng} / \mathrm{mL}(0-34 \mathrm{ng} / \mathrm{mL})$ \\
\hline Cortisol matutino & $0,50 \mu \mathrm{g} / \mathrm{dL}(3-25 \mu \mathrm{g} / \mathrm{dL})$ \\
\hline Anti-21-hidroxilasa & 2,3 U/mL (negativo $<1 \mathrm{U} / \mathrm{mL}$ ) \\
\hline FSH & 44 UI/L (0,3-10 UI/L) \\
\hline $\mathrm{LH}$ & $38 \mathrm{UI} / \mathrm{L}(5-25 \mathrm{UI} / \mathrm{L})$ \\
\hline Estradiol & $28 \mathrm{pg} / \mathrm{mL}(35-400 \mathrm{pg} / \mathrm{mL})$ \\
\hline Vitamina D & $18 \mathrm{ng} / \mathrm{mL}(20-40 \mathrm{ng} / \mathrm{mL})$ \\
\hline PTH & $35,6 \mathrm{ng} / \mathrm{mL}(20-40 \mathrm{ng} / \mathrm{mL})$ \\
\hline Anticardiolipinas lgG & $0,5 \mathrm{glp} / \mathrm{mL}$ (negativo <17 UI GLP) \\
\hline Anticardiolipinas IgM & 0,9 mpl/mL (negativo <15 UI MLP) \\
\hline Beta 2 glicoproteína IgM & 1,1 UI (negativo <20 UI) \\
\hline Beta 2 glicoproteína IgG & 1,6 UI (negativo <20 UI) \\
\hline Coombs directo & Negativo \\
\hline
\end{tabular}

ACTH: corticotropina; CHCM: concentración de hemoglobina corpuscular media; FSH: hormona foliculoestimulante; Ig: inmunoglobulina; LH: hormona luteinizante; PTH: hormona paratiroidea; T4: tiroxina; TSH: tirotropina; UI GLP: unidades internacionales fosfolípidos G; UI MLP: unidades internacionales fosfolípidos M. 
dosis de 2,5 mg a las 4 p. m., con lo que se alcanza una concentración total de 17,5 mg/d repartida en 3 tomas para ajustar a esquema fisiológico acompañado de fludrocortisona tableta en $0,1 \mathrm{mg} / \mathrm{d}$ para corregir el cuadro de hipocortisolismo.

Al tratamiento se añade corticoide tópico (clobetasol en crema) para la corrección de la alopecia areata; también se toman anticuerpos TPO y ATG, ambos positivos, por lo que se considera enfermedad tiroidea inmunológica y se decide continuar la suplencia con levotiroxina.

Se plantea la posibilidad de compromiso gonadal, considerando la amenorrea de 8 meses de evolución, por lo cual se amplían los estudios, que fueron compatibles con falla ovárica prematura en vista del aumento de FSH y LH, hipoestrogenismo y positividad de autoanticuerpos contra enzimas esteroidogénicas, como la 21-hidroxilasa, que es causal de falla suprarrenal aguda e hipogonadismo hipogonadotrófico. Se confirma el cuadro de síndrome poliglandular autoinmunitario tipo II dado por compromiso suprarrenal, tiroideo y gonadal.

\section{Discusión}

El síndrome poliglandular autoinmunitario (SPA) se define como una entidad autoinmunitaria que presenta, como mínimo, dos insuficiencias glandulares en asociación con otras enfermedades inmunológicas no endocrinológicas (1). En la actualidad, se clasifica según los criterios Neufeld y Blizzard con cuatro subtipos clínicos, que tienen órganos blancos, manifestaciones clínicas y picos de presentación diferentes.

Los últimos casos documentados en la literatura sobre síndrome de Schmidt a nivel nacional ocurrieron en Cartagena, Bolívar; se realizó en una serie de casos donde se reportan 5 casos en un periodo de evaluación de 2 años estimando una incidencia de 1,2/100 000 habitantes sin presentar la tríada característica en todos los casos (2).

En el 2018, Barreda y colaboradores brindaron un enfoque de la entidad en el contexto latinoamericano, donde se plantea una incidencia más alta que llega hasta 4,5/100 000 habitantes; a pesar de ello, los casos reportados son escasos, y los países que más han contribuido con información son México, Colombia, Chile y Cuba (3), razón por la cual este aporte se hace más valioso.

La presentación de SPA tipo II se ha asociado a múltiples genes del cromosoma 6 y tiene una asociación importante con el antígeno leucocitario humano (HLA), que en relación con factores ambientales podría desencadenar pérdida de la tolerancia inmunológica y desarrollo del proceso autoinmunitario. El SPA se asocia principalmente a HLADR3, HLADR4, HLA-B8 y a genes del antígeno T linfocítico citotóxico (CTLA4) (3). Se le atribuye herencia autosómica dominante, con baja penetrancia (4).

Generalmente se activan las respuestas tipo $T_{h} 1$ o $T_{h} 2$, que producen autoanticuerpos que pueden depositarse o generar infiltrado inflamatorio crónico de predominio linfocitario gracias a la respuesta inflamatoria generada a expensas de los autoanticuerpos. Además de ello, hay disregulación de las vías de señalización apoptótica, lo que lleva a la destrucción progresiva de los tejidos glandulares con funciones endocrinas (5).

Los síndromes poliglandulares autoinmunitarios son la causa más frecuente de infiltración linfocitaria de las glándulas endocrinas y provocan su destrucción lenta y progresiva, que culmina en insuficiencia hormonal; esto implica que no siempre ocurren las manifestaciones clínicas características de cada padecimiento, ya que dependen del grado y la velocidad de la destrucción glandular (5). La forma de presentación aislada o en conjunto con otras endocrinopatías depende de factores como la diferencia en el tamaño de las glándulas afectadas y la capacidad de renovación de las células endocrinas (6).

El SPA tipo II se caracteriza por la combinación de insuficiencia suprarrenal primaria de base, necesariamente asociada a otra disfunción glandular. Puede recibir el nombre de síndrome de Schmidt, cuando se acompaña de disfunción tiroidea o síndrome de Carpenter, cuando se asocia a disfunción pancreática que se manifiesta con diabetes mellitus tipo 1 (7). Presenta una mayor incidencia en la población adulta, con una relación mujer vs. hombre de 3:1.

El signo más específico de insuficiencia suprarrenal primaria (89-100 \% casos) es la hiperpigmentación de piel y mucosas, que puede llegar a ser difícil de detectar en pacientes con piel bronceada. Generalmente, la pigmentación se presenta como resultado de la elevación plasmática de la propiomelanocortina, precursora de la lipotropina $\beta$, la corticotropina y la melatonina, que afecta principalmente las áreas previamente pigmentadas y expuestas al sol. Asociado a esto se presenta sintomatología por deficiencia mineralocorticoide, como hipotensión ortostática, deseo por la sal y alteraciones hidroelectrolíticas principalmente hiponatremia e hiperpotasemia posterior al compromiso de la corteza suprarrenal (8). La insuficiencia suprarrenal puede confirmarse con una concentración de cortisol matutina menor de $3 \mu \mathrm{g} / \mathrm{dL}$ (83 $\mathrm{nmol} / \mathrm{L}$ ), mientras que concentraciones mayores de $18 \mu \mathrm{g} / \mathrm{dL}$ la excluyen (1). Además de ello, los valores de ACTH realizan la diferenciación entre insuficiencia suprarrenal primaria y secundaria; valores por encima de $100 \mathrm{pg} / \mathrm{mL}$ indican patología primaria, mientras que valores inferiores a este demuestran patología secundaria.

El compromiso tiroideo está presente en el 70-90\% de los casos y genera principalmente tiroiditis de Hashimoto o enfermedad de Graves. Es importante considerar que las manifestaciones clínicas pueden exacerbarse posterior a la suplencia con levotiroxina, posiblemente debido al efecto potenciador de la $\mathrm{T}_{4}$ sobre la función hepática en el metabolismo de los corticosteroides (8). Más del $50 \%$ de los pacientes diabéticos con autoanticuerpos tiroideos progresan a enfermedad autoinmunitaria tiroidea. 
Otro tipo de afecciones pueden presentarse en esta patología, que se consideran como componentes menores de naturaleza autoinmunitaria, dentro de las que destacan el hipogonadismo hipergonadotrópico (11\%), que es la manifestación más importante fuera de la tríada clásica. Es fundamental considerar que se manifiesta con amenorrea primaria, trastornos del desarrollo puberal o falla ovárica prematura que lleva a la disminución o ausencia de secreción esteroidea gonadal, lo que anula el mecanismo de retroalimentación negativa e induce el aumento sérico de LH y FSH, que puede detectarse a través de la toma de estas hormonas y realizando la confirmación de manera histopatológica con gónadas hipoplásicas, fibróticas e infiltradas con linfocitos.

Está descrito que los resultados serológicos positivos para el SPA tipo II preceden al inicio de las manifestaciones (3). Se sugiere solicitar cifras de TSH, $\mathrm{T}_{4}$ libre, $\mathrm{FSH}, \mathrm{LH}$, testosterona, estradiol, glucosa, cortisol matutino, prueba de estimulación con $\mathrm{ACTH}, \mathrm{Na}^{+}, \mathrm{K}^{+}$y $\mathrm{Ca}^{+}$séricos que demuestren la funcionalidad de las glándulas endocrinas, quepueden verse afectadas en esta entidad clínica (5). De manera preventiva, se sugiere realizar tamización a los familiares de primer grado de los pacientes con SPA tipo II, con glucemia en ayunas (3) y en los grupos de riesgo es necesario tamizar también autoanticuerpos y funcionalidad de órganos endocrinos y no endocrinos cada 3 años (6).

Se recomienda manejar la insuficiencia suprarrenal con hidrocortisona de 10 a $12,5 \mathrm{mg} / \mathrm{m}^{2}$ por día; debe administrarse dividida en 2 o 3 dosis para alcanzar esquemas más fisiológicos. En la deficiencia de mineralcorticoide se realiza suplencia con acetato de fludrocortisona, en dosis de 0,05 a 0,2 mg/d (1).
Se confirma que la paciente evaluada tiene insuficiencia suprarrenal primaria, hipotiroidismo autoinmunitario, alopecia areata como manifestación inmunológica de tipo no endocrinológico y manifestaciones de falla ovárica prematura, confirmada a través de paraclínicos; todas estas son compatibles con síndrome de Schmidt. De igual manera, el control periódico en el futuro es de vital importancia, ya que permitirá identificar el compromiso de órganos afectados, pero que en el momento no causan manifestaciones y, por tanto, no han sido identificados.

\section{Conclusión}

El síndrome poliglandular autoinmunitario es una entidad clínica poco frecuente. Posiblemente su abordaje diagnóstico y terapéutico se realice de manera errónea, debido a que su presentación se asume como patologías aisladas. Por tanto, es vital conocer esta condición, sus diversas variantes y enfoques diagnósticos y terapéuticos. Es necesario que después del diagnóstico de cualquier endocrinopatía autoinmunitaria se lleven a cabo controles periódicos y se tenga presente que puede ocurrir la aparición de otra endocrinopatía sobrepuesta.

\section{Conflictos de interés}

Los autores declaran no tener conflicto de interés.

\section{Financiación}

Este artículo no tuvo financiación por parte de entidades públicas o privadas.

\section{Referencias}

1. Navarrete-Tapia U. Síndrome poliglandular autoinmune. Rev Med Hosp Gen Méx. 2013;76(3):143-52

2. Fortich A.,Mora G, Fortich Salvador A,Ramos E síndrome poliglandular autoinmune tipo II en el caribe cartagena colombia Rev.cienc.biomed. 2011; 2 (1): 54-62

3. Barreda-Velit C, Salcedo-pereda R, Ticona J. Síndrome de Schmidt: diagnóstico difícil en el contexto latinoamericano. Rev Med Inst Mex Seguro Social. 2018;56(51):189-93.

4. Resende E, Novoa G, Nascimento M, Loidi L, Saborido R, Cabanas P, et al. Precocious presentation of autoimmune polyglandular syndrome type 2 associated with an AIRE mutation. Hormones. 2015;14(2):312-6.
5. Urbina-Vázquez A, Rodríguez-Lobato E, Aquino-Matus J, Ramírez-Hobak L, Arenas R, Zacarías-Castillo R. Síndrome poliglandular autoinmunitario tipo II (síndrome de Schmidt): Enfermedad de Addison y tiroiditis de Hashimoto asociado con vitíligo. Med Interna Mex. 2017;33(2):241-5.

6. Gürkan E, Çetinarslan B, Güzelmansur İ, Kocabaş B. Latent polyglandular autoimmune syndrome type 2 case diagnosed during a shock manifestation. Gynecol Endocrinol. 2016;32(7):521-3.

7. Zulfiqar AA, Andres E. Association pernicious anemia and autoimmune polyendocrinopathy: a retrospective study. J Med Life. 2017;10(4):250-3.

8. Smith RK, Gerrits PM. A Rare Case of Autoimmune Polyglandular Syndrome Type 2 in a Child with Persistent Fatigue. Global Pediatric Health. 2019;6:1-5. 


\section{Histotal $^{\circledR}$}

Colecalciferol 25.000UI

en ampolla bebible

Nombre Comercial original (Bélgica)
Nombre Comercial en Colombia

Solución Oral

4 Ampollas bebibles

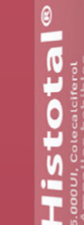

Cada ampolla bebible de Colecalciferol equivalente

\section{Presencia Internacional}

\ Excelente opción para pacientes polimedicados.

3 Puede ser mezclado con cualquier líquido

Mayor adherencia al tratamiento.

Seguridad y eficacia. ${ }^{2}$

$\checkmark$ Vehículo oleoso que brinda mejor absorción y mayor biodisponibilidad. ${ }^{3}$

\&a ingesta mensual es tan eficaz como la ingesta diaria o semanal. ${ }^{4}$

Para toda la familia.

ß Precio Económico.

Material exclusivo para el cuerpo médico

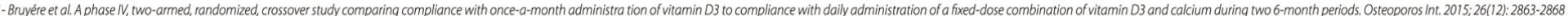

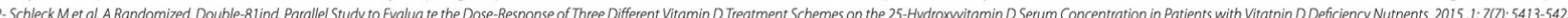

